\title{
Comparative Study between Transforaminal Lumbar Interbody Fusion and Posterolateral Fusion for Treatment of Spondylolisthesis: Clinical Outcomes and Spino-Pelvic Sagittal Balance Parameters
}

\author{
Majid Reza Farrokhi ${ }^{1} \quad$ Keyvan Eghbal ${ }^{2}$ Seyed Reza Mousavi ${ }^{2} \quad$ Mustafa Moumani $^{2}$ \\ Khshayar Bazyari² Bipin chaurasia ${ }^{3}$
}

${ }^{1}$ Department of Neurosurgery, Shiraz University of Medical Sciences, Shiraz, Iran

${ }^{2}$ Shiraz Neurosciences Research Center, Shiraz University of Medical Sciences, Shiraz, Iran

${ }^{3}$ Department of Neurosurgery, Neurosurgery Clinic, Birgunj, Nepal

Indian J Neurotrauma:2021;18:51-58

\begin{abstract}
Address for correspondence Mustafa Moumani, MD, Department of Neurosurgery, Shiraz University of Medical Sciences, Shiraz, Iran (e-mail: trozexa@gmail.com; mustafa.momni@gmail.com).
\end{abstract}

Abstract
Keywords
- spondylolisthesis
- transforaminal lumbar
interbody fusion
- posterolateral fusion
- spino-pelvic sagittal
balance
- low back pain
- clinical outcomes
- radicular pain

Abstract

Objective This retrospective study aims to compare the clinical and radiological outcomes of posterolateral fusion (PLF) with transforaminal lumbar interbody fusion $($ TLIF + PLF) for the treatment of patients with low-grade spondylolisthesis.

Methodology A total of 77 adult patients $\geq 18$ years with low-grade spondylolisthesis, Meyerding grades I and II, were assigned into two groups: 36 patients treated with PLF and 41 patients treated with TLIF + PLF. The PLF group is composed of the patients that were operated with pedicle screw and the TLIF + PLF group is composed of the ones that were operated with fixation and TILF by autografting. Clinical evaluation was performed using the spino-pelvic sagittal balance, Numeric Rating Scale, Oswestry Disability Index, blood loss, operation times, and postoperative hospital stay of the PLF vs TLIF groups. The incidences of postoperative low back pain and radicular pain in the two groups were also recorded. Radiography was performed preoperatively and postoperatively to assess spino-pelvic parameters.

Results Significant restoration of spino-pelvic sagittal balance was observed in the TLIF group after surgery, and all spino-pelvic sagittal balance parameters showed significant improvement in the TLIF group after surgery, while in the PLF group, all spino-pelvic sagittal parameters had improved except the segmental angle lordosis $(p=0.316)$, which showed no significant difference after surgery in the PLF group. Postoperative pelvic incidence and pelvic tilt significantly improved in the TLIF group in comparison to PLF groups. Hence, TLIF can achieve better postoperative spino-pelvic sagittal balance parameters than PLF. There was no difference in the complication rates for each group. Both groups achieved significant improvement in postoperative clinical outcomes, and there was no significant difference in the incidence of postoperative low back pain or radicular pain between the two groups.

Conclusion Both surgical procedures PLF and TLIF were effective. PLF and TLIF can result in improved clinical and radiological outcomes for patients treated for low-grade spondylolisthesis. TLIF can achieve better restoration of spino-pelvic sagittal balance parameters than PLF alone. published online

November 2, 2020
DOI https://doi.org/

$10.1055 / \mathrm{s}-0040-1718781$

ISSN 0973-0508.
(C)2020. Neurotrauma Society of India.

This is an open access article published by Thieme under the terms of the Creative Commons Attribution-NonDerivative-NonCommercial-License, permitting copying and reproduction so long as the original work is given appropriate credit. Contents may not be used for commercial purposes, or adapted, remixed, transformed or built upon. (https://creativecommons.org/licenses/by-nc-nd/4.0/).

Thieme Medical and Scientific Publishers Pvt. Ltd. A-12, 2nd Floor, Sector 2, Noida-201301 UP, India 


\section{Introduction}

Spondylolisthesis is a stress injury, which can be found in patients with chronic low back pain (CLBP) and radicular pain during the clinical assessment. ${ }^{1,2}$ Spondylolisthesis is known as an anatomical defect, commonly seen in the lower lumbar vertebrae. ${ }^{1,2}$ It is the anterior displacement of one vertebra relative to the subjacent vertebra. This defect mostly happens at levels L4-L5 and L5-S1. ${ }^{3}$ In low-grade spondylolisthesis, the spino-pelvic parameters change to compensate for the variations of spine mobility. ${ }^{4}$ Diminishing of the lumbar lordosis (LL) is followed by a reversed ratio of extensors/ flexors muscle power compared with normal controls. ${ }^{5}$ It has been demonstrated that the segmental and global LL are reduced resulting in increased pelvic incidence (PI) and thus transferring the tension to the posterior elements resulting in aggravated deformity, pain, and disability. ${ }^{6}$ Thus, evaluation and measurement of spino-pelvic parameters are recommended before surgical correction of the low-grade spondylolisthesis. ${ }^{4}$ For patients with low-grade spondylolisthesis, nonsurgical treatment that is a combination of pain medication and physiotherapy is recommended. ${ }^{7}$ Within the last decades, posterolateral fusion (PLF) ${ }^{8,9}$ and transforaminal lumbar interbody fusion (TLIF) ${ }^{10,11}$ have become the most popular techniques that can stabilize the lumbar spine, and effectively improve postoperative performance scores. ${ }^{8,9,12,13}$ However, the desirability of each technique is debatable. Several studies compare the use of PLF and TLIF. Potter et al claimed that TLIF is an effective way to achieve lumbar fusion. ${ }^{41}$ The results showed that the pain among $29 \%$ of patients had completely diminished, and in $50 \%$ of patients pain had significantly improved. ${ }^{14}$ Additionally, TLIF has been stated as the most beneficial technique to repair the height of disc space. ${ }^{14}$ On the other hand, Aygün et al confirmed that the complication in the PLF technique was very low. They also reported that the other advantages of this method comprise low blood loss and reduced operation time. ${ }^{15}$ Hence, this retrospective cross-sectional study aims to assess the clinical and radiological outcomes of PLF with TLIF in the treatment of spondylolisthesis.

\section{Materials and Methods}

\section{Study Population}

This retrospective cross-sectional study was performed on a total of 77 patients who underwent PLF or TLIF surgery technique to treat spondylolisthesis during a 3-year period from January 2016 to December 2018 in Neurosurgery Department of Shahid Chamran Hospital, Shiraz University of Medical Sciences, Shiraz, Iran. Thirty-six patients ( 3 men and 33 women) were operated by the PLF technique, while 41 patients ( 16 men and 25 women) underwent TLIF surgery. The present study was approved by the local Ethics Committee of Shiraz University of Medical Sciences. Inclusion criteria were as follows: adult ( $\geq 18$ years) patients with low-grade degenerative or isthmic spondylolisthesiswhere low-grade spondylolisthesis was defined as Meyerding classification grades I and II-who had level 1 spondylolisthesis, and complained of neurological symptoms. The follow-up was at least 12 months, and the parameters and the pain intensity were measured. Exclusion criteria were patients who had more than level 1 spondylolisthesis, and also patients with trauma, tumor, or infection, which is the cause of spondylolisthesis.

\section{Radiographic Data}

A radiograph image of standing anterior-posterior and lateral lumbar spines were taken before and after the surgery. Spinopelvic sagittal balance measurement is defined by Cobb's method. LL is defined as the angle between the superior endplate of L1 vertebrae and the sacral plate. Segmental lordosis $(\mathrm{SL})$ is defined as the angle between the upper endplate of the superior vertebral body and the lower endplate of the inferior vertebral body of the spondylolisthesis level. Sacral slope (SS) is defined as the angle between the sacral plate and the horizontal line. PI is defined by the line perpendicular to the midpoint of the sacral plate and the line between the midpoint of the sacral plate and the center of femoral heads. Pelvic tilt (PT) is defined by the angle between the line connecting the midpoint of the sacral plate with the center of femoral heads and the vertical line ( $\mathbf{- F i g s .} \mathbf{1}$ and $\mathbf{2}$ ).

\section{Classification Systems}

In the current study, spondylolisthesis is classified according to Wiltse and Meyerding classifications that are the most common in this field.

\section{Dynamic Imaging}

All patients had a radiographic assessment of the lumbosacral spine, including standing neutral, flexion, and extension plain lumbar radiography, computed tomography (CT) imaging, and magnetic resonance imaging (MRI). A comparison of flexion/ extension lumbosacral X-ray with lateral X-ray has been traditionally helpful in identifying segmental mobility. ${ }^{16} \mathrm{MRI}$ or CT, when MRI is contraindicated, has become the routine method to assess the degree of stenosis in spondylolisthesis. Hence, it might be helpful in mobility assessment. In comparison to the standing lateral radiography, it is often sufficient to identify mobility in listhetic level, thereby negating the need for flexion/ extension radiographs. ${ }^{17}$
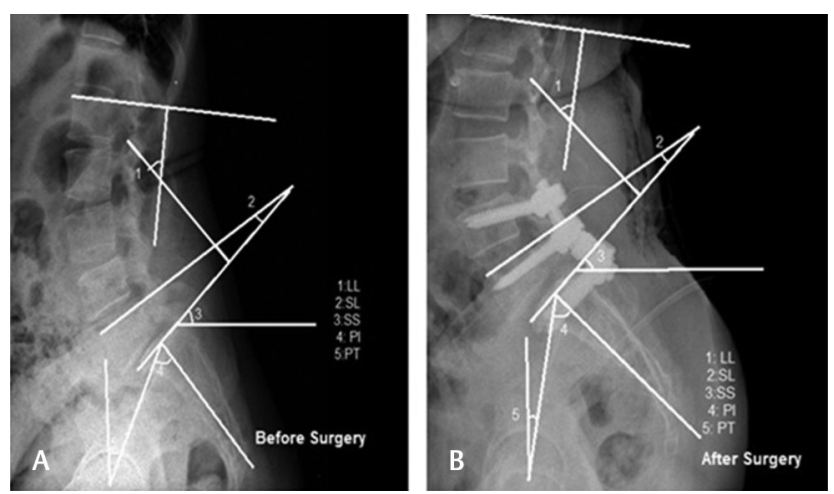

Fig. 1 Spino-pelvic sagittal balance measurement, before surgery (A) and after surgery (B), of a 54-year-old female patient who suffered from isthmic spondylolisthesis at the L5-S1 level and underwent posterolateral fusion (PLF). LL, lumber lordosis; SL, segmental lordosis; SS, sacral slope; PI, pelvic incidence; PT, pelvic tilt. 

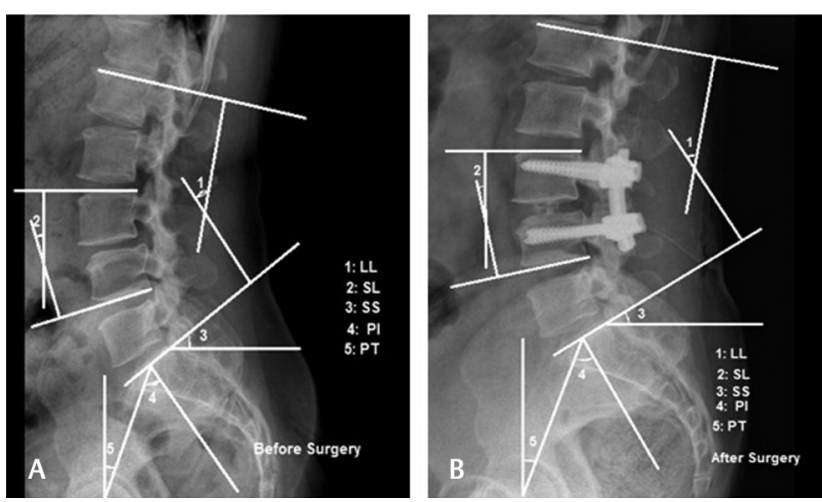

Fig. 2 Spino-pelvic sagittal balance measurement, before surgery (A) and after surgery (B), of a 51-year-old female patient who suffered from degenerative spondylolisthesis at the L3-L4 level and underwent transforaminal lumbar interbody fusion (TLIF). LL, lumber lordosis; SL, segmental lordosis; SS, sacral slope; PI, pelvic incidence; PT, pelvic tilt.

\section{Stability Assessment}

We defined instability as a radiologically demonstrated motion of the spondylolisthesis beyond the expected normal values in a normal spine. There are many other classifications, as White and Panjabi considered sagittal plane displacement $>4.5 \mathrm{~mm}$ (or $15 \%$ of the anteroposterior diameter of the vertebral body) or sagittal plane angulation $>22$ degrees to be potentially unstable, while Nachemson mentioned instability to be $>3 \mathrm{~mm}$ translational motion and $>10$ degrees angular motion between L1 and L5 and $>4 \mathrm{~mm}$ translational motion and $>20$ degrees angular motion at L5-S1. ${ }^{18}$ Although the degenerative spondylolisthesis is not always unstable, the identification of the patients with dynamic instability can help manage this situation. ${ }^{19}$

\section{Operative Technique}

The patients were all operated under the general anesthesia in the prone position. Two rolls were placed under the chest and the pelvis to avoid pressure injury during the operation. The posterior approach was utilized and the levels were cleared using intraoperative fluoroscopy. A midline incision was made to expose the spinous processes by exposing the posterolateral elements. Posterior spinal fixation was performed via pedicular screw insertion (SA-IRAN/ ATA/OSVEH set) in all patients. Pedicular screw fixation of the involved levels was performed under the guidance of anatomical landmarks and fluoroscopy using the polyaxial screws. In the PLF group, a bone graft from the spinous processes and laminae was placed on decorticated transverse processes and facet joints bilaterally. Laminectomy and root foraminotomy were performed according to the side of the patients' complement. In the TLIF group, a nearly complete discectomy and endplate decortications were performed. Intervertebral disc space spreaders were then inserted and rotated to restore the normal disc space height. Bone graft from the lamina and spinous processes was placed into the cage before insertion. Cages (SA-IRAN/ ATA/OSVEH set) packed with bone graft were inserted into the disc space. Next, the cage was filled with autograft and allograft (Tissue Regeneration Inc., Kish, Iran) and was then carefully impacted into position. The cage was pushed as anterior as possible and the location was checked with fluoroscopy. Complete evaluation of dural sac and neural foramina was done, and acceptable decompression was achieved. Irrigation was done, and gelfoam was applied over the dura. Bilateral PLF with transverse process osteotomy and allograft was done. The wound was copiously irrigated and closed with a Hemovac drain in place (-Figs. 3 and $\mathbf{4}$ ).

\section{Clinical Outcomes}

To assess the clinical outcomes before and after surgery, the following two methods were used.

1. Oswestry Disability Index (ODI): This questionnaire was designed in a way that one can manage how back pain affects the daily life of a patient. This questionnaire consists of 10 parts. The total score for each section is 5 points. ${ }^{20}$

2. Numeric Rating Scale (NRS): NRS requires the patient to rate their pain on a scale from 0 to 10 , where 0 is assigned to no pain and 10 is the worst pain imaginable. ${ }^{21}$

\section{Statistical Analysis}

For data analysis, Statistical Package for the Social Sciences (SPSS Inc.; Chicago, Illinois, United States) version 15 was used. We analyzed the dependent variable as a mean \pm standard deviation (SD). Independent $t$-test and chi-squared test were used to analyze the categorical variables. $p$-Values less than 0.05 were considered to be statistically significant.

\section{Results}

- Table 1 shows the demographic characteristics of the patients in PLF and TLIF groups. A total of 77 patients (36 patients treated with PLF and 41 patients treated with TLIF) participated in this study. The average ages of patients in PLF and TLIF groups were $55.34 \pm 8.76$ and $52.02 \pm 7.95$ years. In addition to the descriptive level, the findings revealed that hospital stay (days), follow-up (months), operative time (hour), and blood loss $(\mathrm{mL})$ means and SD were $7.58 \pm 3.23,17.88 \pm 7.31,4.6 \pm 0.898$, and $893.05 \pm 399.13$ in the PLF group and $8.24 \pm 2.32,19.80 \pm 8.73,4.31 \pm 0.723$, and $1,073.65 \pm 120.82$ in the TLIF group, respectively.

All parameters of the spino-pelvic sagittal balance, including LL ( $p=0.002)$, SS ( $p=0.007)$, PI $(p<0.001)$, and PT $(p=0.04)$ in the PLF group and $\operatorname{LL}(p=0.007), \operatorname{SL}(p=0.006)$, SS $(p<0.001)$, PI $(p<0.001)$, and PT $(p<0.001)$ in the TLIF group significantly improved after surgery. However, $\operatorname{SL}(p=0.316)$ was not found to be statistically different from the baseline in the PLF group after surgery. Almost all patients in both groups achieved significant improvements throughout NRS $(p<0.001)$ and ODI $(p<0.001)$ after surgery. The changes in spino-pelvic parameters and the clinical outcome measures in PLF and TLIF groups are summarized in - Tables $\mathbf{2}$ and $\mathbf{3}$.

- Table 4 shows the comparative assessment of the variables for any spino-pelvic sagittal balance between PLF 

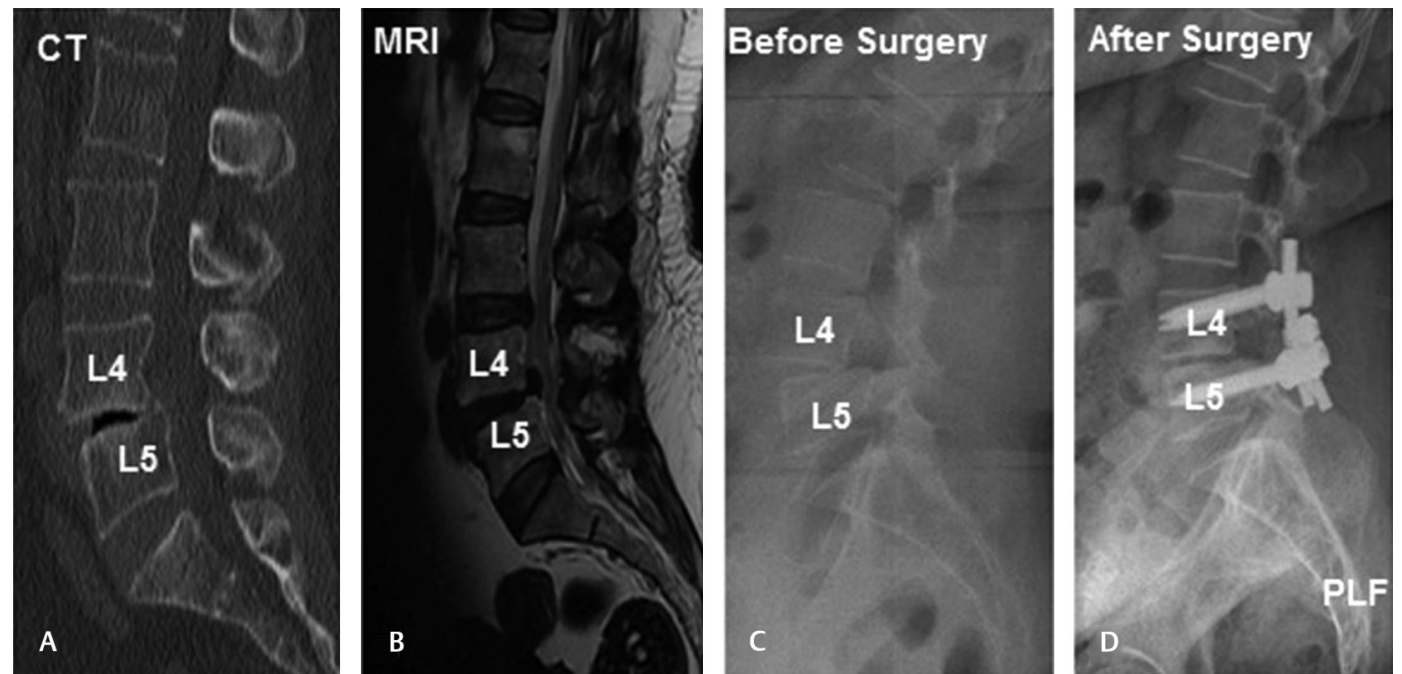

Fig. 3 Preoperative sagittal-computed tomographic scan (A), sagittal T2-weighted magnetic resonance image (B), sagittal lateral view (C), and postoperative sagittal lateral view (D) of a 52-year-old female patient who suffered from degenerative spondylolisthesis at the L4-L5 level and underwent posterolateral fusion (PLF). CT, computed tomography; MRI, magnetic resonance imaging.
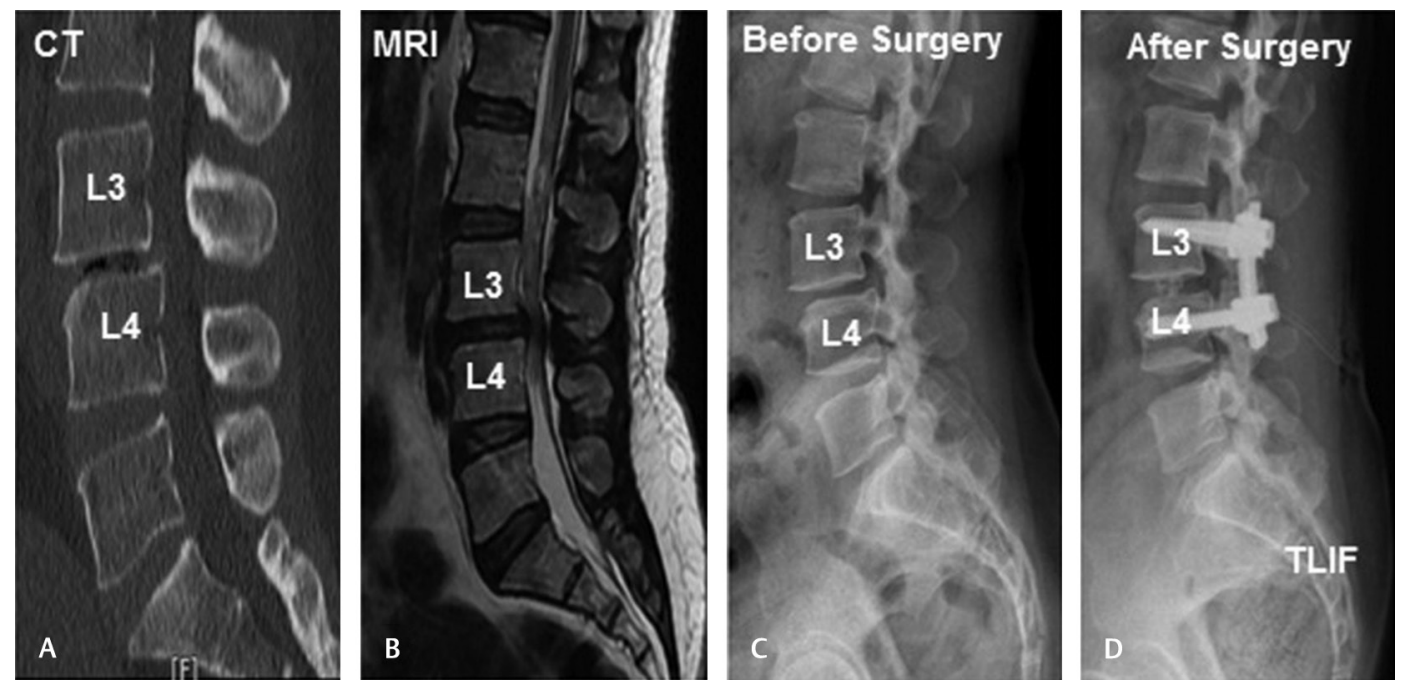

Fig. 4 Preoperative sagittal-computed tomographic scan (A), sagittal T2-weighted magnetic resonance image (B), sagittal lateral view (C), and postoperative sagittal lateral view (D) of a 51 -year-old female patient who suffered from degenerative spondylolisthesis at the L3-L4 level and underwent transforaminal lumbar interbody fusion (TLIF). CT, computed tomography; MRI, magnetic resonance imaging.

and TLIF groups. No significant differences were observed between the two groups preoperatively, but the results of the postoperative level showed that PI $(p=0.023)$ and PT $(p=0.021)$ in the PLF group were significantly different from the TLIF group.

Finally, 8/36 (22.22\%) patients in the PLF group and 10/41 (24.39\%) in the TLIF group suffered from low back pain postoperatively. Besides, 11/36 (30.55\%) and 13/41 (31.70\%) patients in the PLF and TLIF groups were experiencing radicular pain, respectively. The comparison of clinical outcome measures between PLF and TLIF groups is summarized in - Table 5.

Overall, we recorded two patients with complications. One of them was in the TLIF group $(1 / 41 ; 2 \%)$ who developed postoperative epidural hematoma presenting as back pain and paraparesis 24 hours after the operation. The patient underwent emergency reoperation and the hematoma was removed and he recovered partially after 4 months of physiotherapy and conservative management. Besides, one patient was in the PLF group $(1 / 36 ; 2 \%)$ who developed ipsilateral weakness of extensor hallucis longus. The muscle power recovered completely after 3 months of physiotherapy and conservative care.

\section{Discussion}

Few studies have directly compared the TLIF and PLF techniques. Nevertheless, a systematic review and meta-analysis by Levin et al reported that in patients with spondylolisthesis, TLIF is superior to PLF. This finding is the result of radiographic data. However, the data from this study support and 
Table 1 Comparative analysis of demographics of the two groups PLF and TLIF

\begin{tabular}{|c|c|c|c|}
\hline & PLF group & TLIF group & $p$-Value ${ }^{a}$ \\
\hline Number of patients & 36 & 41 & \\
\hline Sex (male/female) & $3 / 33$ & $16 / 25$ & 0.002 \\
\hline Age & $(55.34 \pm 8.76)$ & $(52.02 \pm 7.95)$ & 0.088 \\
\hline \multicolumn{4}{|l|}{ Operation segments } \\
\hline L3-L5 & 18 & 23 & \multirow[t]{4}{*}{0.985} \\
\hline L4-S1 & 1 & 0 & \\
\hline L4-L5 & 1 & 3 & \\
\hline L5-S1 & 5 & 15 & \\
\hline Hospital stays (days) & $(7.58 \pm 3.23)$ & $(8.24 \pm 2.32)$ & 0.039 \\
\hline Follow-up (months) & $(17.88 \pm 7.31)$ & $(19.80 \pm 8.73)$ & 0.304 \\
\hline Operative times (hour) & $(4.6 \pm 0.898)$ & $(4.31 \pm 0.723)$ & 0.029 \\
\hline Blood loss (mL) & $(893.05 \pm 399.13)$ & $(1,073.65 \pm 120.82)$ & 0.0001 \\
\hline
\end{tabular}

Abbreviations: PLF, posterolateral fusion; TLIF, transforaminal lumbar interbody fusion. a $p<0.05$.

Table 2 Changes in spino-pelvic sagittal balance parameters NRS and ODI in PLF group

\begin{tabular}{|l|l|l|l|}
\hline & Before surgery & After surgery & $p_{\text {-Value }}$ \\
\hline LL & $(48.47 \pm 9.62)$ & $(49.19 \pm 8.86)$ & 0.002 \\
\hline SL & $(14.47 \pm 4.98)$ & $(14.69 \pm 6.10)$ & 0.316 \\
\hline SS & $(30.36 \pm 9.15)$ & $(31.77 \pm 6.90)$ & 0.007 \\
\hline PI & $(53.83 \pm 9.46)$ & $(54.16 \pm 7.97)$ & 0.0001 \\
\hline PT & $(22.47 \pm 6.00)$ & $(22.50 \pm 6.51)$ & 0.044 \\
\hline NRS & $(6.88 \pm 1.76)$ & $(4.61 \pm 1.88)$ & 0.0001 \\
\hline ODI & $(48.00 \pm 5.32)$ & $(27.36 \pm 7.10)$ & 0.0001 \\
\hline
\end{tabular}

Abbreviations: LL, lumbar lordosis; NRS, Numeric Rating Scale; ODI, Oswestry Disability Index; PI, pelvic incidence; PLF, posterolateral fusion; PT, pelvic tilt; SL, segmental lordosis; SS, sacral slope.

${ }^{\mathrm{a}} p<0.05$.

Table 3 Changes in spino-pelvic sagittal balance parameters NRS and ODI in TLIF group

\begin{tabular}{|l|l|l|l|}
\hline & Before surgery & After surgery & $p$-Value \\
\hline LL & $(48.65 \pm 8.73)$ & $(47.317 \pm 8.44)$ & 0.007 \\
\hline SL & $(15.19 \pm 6.20)$ & $(16.95 \pm 5.77)$ & 0.006 \\
\hline SS & $(33.26 \pm 7.15)$ & $(32.07 \pm 5.58)$ & 0.0001 \\
\hline PI & $(52.14 \pm 7.39)$ & $(50.60 \pm 5.38)$ & 0.0001 \\
\hline PT & $(18.46 \pm 7.22)$ & $(19.26 \pm 5.54)$ & 0.0001 \\
\hline NRS & $(7.48 \pm 1.66)$ & $(3.80 \pm 1.43)$ & 0.0001 \\
\hline ODI & $(50.09 \pm 6.62)$ & $(24.58 \pm 6.66)$ & 0.0001 \\
\hline
\end{tabular}

Abbreviations: LL, lumbar lordosis; NRS, Numeric Rating Scale; ODI, Oswestry Disability Index; PI, pelvic incidence; PT, pelvic tilt; SL, segmental lordosis; SS, sacral slope; TLIF, transforaminal lumbar interbody fusion. ${ }^{\mathrm{a}} \mathrm{p}<0.05$.

only confirm the superiority of TLIF over PLF. ${ }^{22}$ PLF is considered superior to the other fusion procedures in terms of anterior column support, sufficient decompression of nerve roots, and restoration of lumbar alignment. ${ }^{23,24}$ Several previous studies reported the improvement in clinical efficacy of PLF. 25

In our study, the results showed that both surgical procedures, PLF and TLIF, were effective. However, the superiority of each method is still debatable. It can be said that the most common interbody fusion technique is TLIF, whereas PLF is the most common technique for treating lumbar spondylolisthesis. ${ }^{26}$ In our previous study in 2012, we evaluated PLF with posterior lumbar interbody fusion (PLIF) in isthmic spondylolisthesis patients. The results showed that PLF had improved clinical outcomes and obtained much less back pain than PLIF. In the current study, significant restoration of 
Table 4 Comparison of spino-pelvic sagittal balance parameters between two groups PLF and TLIF

\begin{tabular}{|l|l|l|l|}
\hline & PLF $(\boldsymbol{n}=\mathbf{3 6})$ & TLIF $(\boldsymbol{n}=41)$ & $p$-Value \\
\hline LL (before surgery) & $(48.47 \pm 9.62)$ & $(48.65 \pm 8.73)$ & 0.929 \\
\hline LL (after surgery) & $(49.19 \pm 8.86)$ & $(47.317 \pm 8.44)$ & 0.352 \\
\hline SL (before surgery) & $(14.47 \pm 4.98)$ & $(15.19 \pm 6.20)$ & 0.578 \\
\hline SL (after surgery) & $(14.69 \pm 6.10)$ & $(16.95 \pm 5.77)$ & 0.983 \\
\hline SS (before surgery) & $(30.36 \pm 9.15)$ & $(33.26 \pm 7.15)$ & 0.123 \\
\hline SS (after surgery) & $(31.77 \pm 6.90)$ & $(32.07 \pm 5.58)$ & 0.836 \\
\hline PI (before surgery) & $(53.83 \pm 9.46)$ & $(52.14 \pm 7.39)$ & 0.383 \\
\hline PI (after surgery) & $(54.16 \pm 7.97)$ & $(50.60 \pm 5.38)$ & 0.023 \\
\hline PT (before surgery) & $(22.47 \pm 6.00)$ & $(18.46 \pm 7.22)$ & 0.010 \\
\hline PT (after surgery) & $(22.50 \pm 6.51)$ & $(19.26 \pm 5.54)$ & 0.021 \\
\hline
\end{tabular}

Abbreviations: LL, lumbar lordosis; PI, pelvic incidence; PLF, posterolateral fusion; PT, pelvic tilt; SL, segmental lordosis; SS, sacral slope; TLIF, transforaminal lumbar interbody fusion.

${ }^{\mathrm{a}} p<0.05$.

Table 5 Comparison of clinical outcomes between PLF and TLIF groups

\begin{tabular}{|l|l|l|l|}
\hline & PLF $(\boldsymbol{n}=36)$ & TLIF $(\boldsymbol{n}=41)$ & \multirow{2}{*}{$\boldsymbol{p}$-Value } \\
\hline NRS (before surgery) & $(6.88 \pm 1.76)$ & $(7.48 \pm 1.66)$ & \multirow{2}{*}{0.0001} \\
\cline { 1 - 2 } NRS (after surgery) & $(4.61 \pm 1.88)$ & $(3.80 \pm 1.43)$ & \\
\cline { 1 - 2 } ODI (before surgery) & $(48.00 \pm 5.32)$ & $(50.09 \pm 6.62)$ & \\
\cline { 1 - 2 } ODI (after surgery) & $(27.36 \pm 7.10)$ & $(24.58 \pm 6.66)$ & \\
\hline CLBP & $(8 / 36)$ & $(10 / 41)$ & \\
\hline Radicular pain & $(11 / 36)$ & $(13 / 41)$ & \\
\hline
\end{tabular}

Abbreviations: CLBP, chronic low back pain; NRS, Numeric Rating Scale; ODI, Oswestry Disability Index; PLF, posterolateral fusion; TLIF, transforaminal lumbar interbody fusion.

${ }^{\mathrm{a}} \mathrm{p}<0.05$.

all spino-pelvic sagittal parameters was observed in the TLIF group after surgery, and, at the same time, all spino-pelvic sagittal parameters had improved in the PLF group, except the SL, which showed no significant difference after surgery in the PLF group. Previous studies have revealed that deterioration of natural spino-pelvic sagittal balance may lead to poor clinical outcomes. It was also reported that patients having low back pain showed lower SS and LL compared with normal controls. ${ }^{27}$ However, the influence of spino-pelvic sagittal balance restoration on clinical outcomes remains controversial. ${ }^{28,29}$ Although most patients were satisfied with the clinical results after surgery, some patients suffered from newly initiated or recurrent low back pain, which is called failed surgery syndrome or chronic low back pain. ${ }^{30}$ Wang et al stated that paraspinal muscle degeneration and low back pain before surgery are risk factors for CLBP after surgery. ${ }^{31}$ Mukai et al reported that postoperative muscle strain may be related to low back pain. ${ }^{32}$ There was no difference in the rate of complication in each group. Both groups achieved significant improvement postoperatively in the clinical outcomes, and there was no significant difference in the incidence of postoperative low back pain or radicular pain between the two groups. The results of this study showed that postoperatively, 8/36 (22.22\%) patients in the PLF group and $10 / 41$ (24.39\%) patients in the TLIF group suffered from low back pain. Furthermore, 11/36 (30.55\%) and $13 / 41$ (31.70\%) patients in PLF and TLIF groups, respectively, were experiencing radicular pain.

The comparison between two techniques on the same number of 30 patients was conducted by Challier et al. ${ }^{33}$ The 2-year follow-up of the patients showed that both techniques resulted in a similar clinical outcome as the visual analog scale scores for back pain were improved by 3.8 and 3.3 in PLF and TLIF groups, respectively. Accordingly, 57\% of the patients (17 out of 30 ) were fused in the PLF group, whereas the percentage was $97 \%$ (29 out of 30) for the TLIF one. Etemadifar et al conducted a randomized controlled trial in 2016 to compare the PLF $(n=25)$ and TLIF $(n=25)$ techniques for all posterior lumbar spondylolisthesis scores (grades 1, 2, and 3 ). The data showed that $72 \%, 26 \%$, and $2 \%$ of patients had grades 1,2 , and 3 , respectively. The TLIF group reported lower average scores at 24-month follow-up for back pain, leg pain, and ODI $(p<0.05)$. Also, in the ODI assessment in the PLF group, 53.2\% had pain improvement compared with TLIF group (56.7\%). ${ }^{34}$ In this study, both TLIF and PLF achieved the appropriate restoration of spinal alignment, with no significant differences in the clinical outcomes and complications rate. Furthermore, clinical outcomes (NRS and ODI) had improved significantly after surgery in both PLF and TLIF groups. Potter et al stated that TLIF is an effective way 
to reach lumbar fusion. The results of these studies showed that pain among $29 \%$ of patients had completely diminished, and $50 \%$ of patients had significantly improved their pain. ${ }^{34} \mathrm{~A}$ further study by Hackenberg et al in 2005 also showed that by the TLIF technique, there is a significant reduction in ODI score, $8 \%$, while it reached $89 \%$ fusion rate. ${ }^{35}$

In an observational study, Carreon et al also compared the clinical improvement in 101 patients with these techniques and the results suggested that visual analog scale scores for back and leg pains improved by 4.2 and 4.6 respectively for TLIF group and 3.5 and 3.7 for PLF group. ${ }^{36}$

TLIF exhibited a significantly higher incidence of adjacent segment disease. Fixing intervertebral cages or grafts can increase stress on adjacent sections by increasing segmental rigidity, subsequently accelerating the degenerative process of adjacent segments after surgery. Previous studies showed that intervertebral fusion does not have a significant effect on global clinical results after surgery. ${ }^{37,38}$ Imbalance of the lumbar and pelvis may be a potential cause for degenerative lumbar diseases. Ferrero et al conducted a study on 654 patients and found that degenerative spondylolisthesis is relevant to a large PI and small LL. ${ }^{5}$ This result is in agreement with another study, ${ }^{39}$ which indicated that imbalanced sagittal parameters are formed gradually during the process of lumbar degeneration.

Radovanovic et al showed that patients with poor spino-pelvic sagittal balance restoration always have poor clinical outcome.$^{40}$ In this study, postoperative PI and PT improved significantly in the TLIF group in comparison to the PLF group. These results indicate that the added cages or grafts in the TLIF group were able to restore the pelvic sagittal balance with improvements in PI and PT and counteract the worsening of lumbosacral sagittal balance parameters. Hence, it can achieve better postoperative spino-pelvic sagittal balance parameters. However, PLF cannot offer sufficient support to improve the abnormal sagittal balance parameters.

\section{Limitation}

In this study we had some limitations, such as the number of patients was too small, and the follow-up period was limited; we also included those with 1 year of follow-up while most studies have included those with at least 2 years. For evaluation of the fusion rate, we need to perform a high-resolution thin-cut CT-scan, which was not performed in the current study. Therefore, no data could be collected for the fusion rate in these patients and groups. Our study was a retrospective cross-sectional study but not randomized. And the cause of CLBP after surgery was investigated due to short follow-up. Hence, cohort studies are recommended in the future.

\section{Conclusion}

PLF and TLIF can improve the clinical and radiologic outcomes of patients. However, TLIF can accomplish better restoration of spino-pelvic sagittal balance parameters in comparison to PLF alone.

\section{Conflict of Interest}

None declared.

\section{Acknowledgment}

The authors would like to thank all the patients and their families who participated in this study.

\section{References}

1 Selhorst M, Allen M, McHugh R, MacDonald J. Rehabilitation considerations for spondylolysis in the youth athlete. Int J Sports Phys Ther 2020;15(2):287-300

2 Aoki Y, Takahashi H, Nakajima A, et al. Prevalence of lumbar spondylolysis and spondylolisthesis in patients with degenerative spinal disease. Sci Rep 2020;10(1):6739

3 Iii WS, Orías AA, Shifflett GD, et al. Image-based markers predict dynamic instability in lumbar degenerative spondylolisthesis. Neurospine 2020;17(1):221-227

4 Faraj SS, De Kleuver M, Vila-Casademunt A, et al. Sagittal radiographic parameters demonstrate weak correlations with pretreatment patient-reported health-related quality of life measures in symptomatic de novo degenerative lumbar scoliosis: a European multicenter analysis. J Neurosurg Spine 2018;28(6):573-580

5 Ferrero E, Ould-Slimane M, Gille O, Guigui P; French Spine Society (SFCR). Sagittal spinopelvic alignment in 654 degenerative spondylolisthesis. Eur Spine J 2015;24(6):1219-1227

6 Kepler CK, Hilibrand AS, Sayadipour A, et al. Clinical and radiographic degenerative spondylolisthesis (CARDS) classification. Spine J 2015;15(8):1804-1811

7 Farrokhi MR, Rahmanian A, Masoudi MS. Posterolateral versus posterior interbody fusion in isthmic spondylolisthesis. J Neurotrauma 2012;29(8):1567-1573

8 Endler P, Ekman P, Möller H, Gerdhem P. Outcomes of posterolateral fusion with and without instrumentation and of interbody fusion for isthmic spondylolisthesis: a prospective study. J Bone Joint Surg Am 2017;99(9):743-752

9 Gad Abdelkader S, El Zahlawy HN, Elkhateeb TM. Interbody fusion versus posterolateral fusion in treatment of low grade lytic spondylolisthesis. Acta Orthop Belg 2019;85(3):269-273

10 Wu A-M, Hu ZC, Li XB, et al. Comparison of minimally invasive and open transforaminal lumbar interbody fusion in the treatment of single segmental lumbar spondylolisthesis: minimum two-year follow up. Ann Transl Med 2018;6(6):105

11 Peng $\mathrm{P}$, Chen $\mathrm{K}$, Chen $\mathrm{H}$, et al. Comparison of $\mathrm{O}$-arm navigation and microscope-assisted minimally invasive transforaminal lumbar interbody fusion and conventional transforaminal lumbar interbody fusion for the treatment of lumbar isthmic spondylolisthesis. J Orthop Translat 2019;20:107-112

12 Pooswamy S, Muralidharagopalan NR, Subbaiah S. Transforaminal lumbar interbody fusion versus instrumented posterolateral fusion in Grade I/II spondylolisthesis. Indian J Orthop 2017;51(2):131-138

13 Eghbal K, Pourabbas B, Abdollahpour HR, Mousavi R. Clinical, functional, and radiologic outcome of single-and double-level transforaminal lumbar interbody fusion in patients with low-grade spondylolisthesis. Asian J Neurosurg 2019;14(1):181-187

14 Dehoux E, Fourati E, Madi K, Reddy B, Segal P. Posterolateral versus interbody fusion in isthmic spondylolisthesis: functional results in 52 cases with a minimum follow-up of 6 years. Acta Orthop Belg 2004;70(6):578-582

15 Aygün H, Cakar A, Hüseyinoğlu N, Hüseyinoğlu U, Celik R. Clinical and radiological comparison of posterolateral fusion and posterior interbody fusion techniques for multilevel 
lumbar spinal stabilization in manual workers. Asian Spine J 2014;8(5):571-580

16 Kanayama M, Hashimoto T, Shigenobu K, Oha F, Ishida T, Yamane S. Intraoperative biomechanical assessment of lumbar spinal instability: validation of radiographic parameters indicating anterior column support in lumbar spinal fusion. Spine 2003;28(20):2368-2372

17 Liu N, Wood KB, Schwab JH, et al. Utility of flexion-extension radiographs in lumbar spondylolisthesis: a prospective study. Spine 2015;40(16):E929-E935

18 Nachemson A. The role of spine fusion: question 8. Spine 1981;6(3):306-307

19 Hasegawa K, Kitahara K, Shimoda H, et al. Lumbar degenerative spondylolisthesis is not always unstable: clinicobiomechanical evidence. Spine 2014;39(26):2127-2135

20 Fairbank JC, Pynsent PB. The Oswestry disability index. Spine 2000;25(22):2940-2952, discussion 2952

21 Jensen MP, Karoly P, Self-report scales and procedures for assessing pain in adults. In: Turk DC, Melzack R, eds. Handbook of Pain Assessment. New York, NY: The Guilford Press; 2001:15-34

22 Levin JM, Tanenbaum JE, Steinmetz MP, Mroz TE, Overley SC. Posterolateral fusion (PLF) versus transforaminal lumbar interbody fusion (TLIF) for spondylolisthesis: a systematic review and meta-analysis. Spine J 2018;18(6):1088-1098

23 Chen YC, Zhang L, Li EN, Ding LX, Zhang GA, Hou Y, Yuan W. Comparison of posterolateral fusion and posterior lumbar interbody fusion in the treatment of lumbar spondylolisthesis: a meta-analysis. J Invest Surg 2019;32(4):290-297

24 Farrokhi MR, Yadollahikhales G, Gholami M. Mousavi SR, Mesbahi AR, Asadi-Pooya AA. Clinical outcomes of posterolateral fusion vs. posterior lumbar interbody fusion in patients with lumbar spinal stenosis and degenerative instability. Pain Physician 2018;21(4):383-406

25 Zhang S, Ye C, Lai Q et al. Double-level lumbar spondylolysis and spondylolisthesis: a retrospective study. J Orthop Surg Res 2018;13(1):55

26 Glassman SD, Carreon LY, Ghogawala Z, Foley KT, McGirt $\mathrm{MJ}$, Asher AL. Benefit of transforaminal lumbar interbody fusion vs posterolateral spinal fusion in lumbar spine disorders: a propensity-matched analysis from the National Neurosurgical Quality and Outcomes Database Registry. Neurosurgery 2016;79(3):397-405

27 Chaléat-Valayer E, Mac-Thiong JM, Paquet J, Berthonnaud E, Siani F, Roussouly P. Sagittal spino-pelvic alignment in chronic low back pain. Eur Spine J 2011;20(5, Suppl 5):634-640

28 Bourghli A, Aunoble S, Reebye O, Le Huec JC. Correlation of clinical outcome and spinopelvic sagittal alignment after surgical treatment of low-grade isthmic spondylolisthesis. Eur Spine J 2011;20(5, Suppl 5):663-668

29 Natale M, D’Oria S, Nero VV, Squillante E, Gentile M, Rotondo M. Long-term effects of intrathecal baclofen in multiple sclerosis. Clin Neurol Neurosurg 2016;143:121-125
30 Schaller B. Failed back surgery syndrome: the role of symptomatic segmental single-level instability after lumbar microdiscectomy. Eur Spine J 2004;13(3):193-198

31 Wang $\mathrm{H}$, Wang $\mathrm{T}$, Wang $\mathrm{Q}$ Ding W. Incidence and risk factors of persistent low back pain following posterior decompression and instrumented fusion for lumbar disk herniation. J Pain Res 2017;10:1019-1025

32 Mukai Y, Takenaka S, Hosono N, Miwa T, Fuji T. Intramuscular pressure of the multifidus muscle and low-back pain after posterior lumbar interbody fusion: comparison of mini-open and conventional approaches. J Neurosurg Spine 2013;19(6):651-657

33 Challier V, Boissiere L, Obeid I, et al. One-level lumbar degenerative spondylolisthesis and posterior approach: is transforaminal lateral interbody fusion mandatory?: A randomized controlled trial with 2-year follow-up. Spine 2017;42(8):531-539

34 Etemadifar MR, Hadi A, Masouleh MF. Posterolateral instrumented fusion with and without transforaminal lumbar interbody fusion for the treatment of adult isthmic spondylolisthesis: a randomized clinical trial with 2-year follow-up. J Craniovertebr Junction Spine 2016;7(1):43-49

35 Hackenberg L, Halm H, Bullmann V, Vieth V, Schneider M, Liljenqvist U. Transforaminal lumbar interbody fusion: a safe technique with satisfactory three to five year results. Eur Spine J 2005;14(6):551-558

36 Carreon LY, Glassman SD, Ghogawala Z, Mummaneni PV, McGirt MJ, Asher AL. Modeled cost-effectiveness of transforaminal lumbar interbody fusion compared with posterolateral fusion for spondylolisthesis using N(2)QOD data. J Neurosurg Spine 2016;24(6):916-921

37 LuoJ, Cao K, YuT, etal. Comparison of posterior lumbar interbody fusion versus posterolateral fusion for the treatment of isthmic spondylolisthesis. Clin Spine Surg 2017;30(7):E915-E922

38 Lee GW, Lee SM, Ahn MW, Kim HJ, Yeom JS. Comparison of posterolateral lumbar fusion and posterior lumbar interbody fusion for patients younger than 60 years with isthmic spondylolisthesis. Spine 2014;39(24):E1475-E1480

39 Rice JW, Sedney CL, Daffner SD, Arner JW, Emery SE, France JC. Improvement of segmental lordosis in transforaminal lumbar interbody fusion: a comparison of two techniques. Global Spine J 2016;6(3):229-233

40 Radovanovic I, Urquhart JC, Ganapathy V, et al. Influence of postoperative sagittal balance and spinopelvic parameters on the outcome of patients surgically treated for degenerative lumbar spondylolisthesis. J Neurosurg Spine 2017;26(4):448-453

41 Potter BK, Freedman BA, Verwiebe EG, Hall JM, Polly Jr DW, Kuklo TR. Transforaminal lumbar interbody fusion: clinical and radiographic results and complications in 100 consecutive patients. Clin Spine Surg 2005;1,18(4):337-346 